\title{
Inhaled Corticosteroids And Risk Of Tuberculosis
}

\section{In Patients With Obstructive Lung Diseases: A Systematic Review And Meta-Analysis Of Non- randomized Studies}

This article was published in the following Dove Press journal:

International Journal of Chronic Obstructive Pulmonary Disease

\section{Giorgio Castellana $\mathbb{( D}^{1, *}$ \\ Marco Castellana $\mathbb{D}^{2, *}$ \\ Carlo Castellana ${ }^{3}$ \\ Giuseppe Castellana ${ }^{4}$ \\ Emanuela Resta ${ }^{5}$ \\ Mauro Carone' \\ Onofrio Resta ${ }^{6}$ \\ 'Pulmonary Division, Istituti Clinici} Scientifici Maugeri SpA SB Pavia, IRCCS Cassano Murge, Bari, Italy; ${ }^{2}$ Department of Emergency and Organ Transplantation, Section of Internal Medicine, Endocrinology, Andrology and Metabolic Diseases, University of Bari Aldo Moro, Bari, Italy;

${ }^{3}$ University of Bari Aldo Moro, Bari, Italy;

${ }^{4}$ Pulmonary Division, District Health Center ASL Bari, Conversano, Italy; ${ }^{5}$ University of Italian Switzerland, Lugano, Switzerland; ${ }^{6}$ Cardio-Thoracic Department, Institute of Respiratory Diseases, University of Bari "Aldo Moro", Bari, Italy

*These authors contributed equally to this work
Correspondence: Giorgio Castellana Pulmonary Division, Istituti Clinici Scientifici Maugeri SpA SB Pavia, IRCCS

Cassano Murge, Cassano Murge, Bari, Italy

Tel +3980 78I 4379

$\mathrm{Fax}+398078 \mid 4308$

Email giorgio.castellana@icsmaugeri.it
Background: An association between systemic corticosteroids and tuberculosis (TB) is reported in the literature. Here within, we conducted a systematic review and meta-analysis to evaluate the effects of inhaled corticosteroids (ICS) on the risk of TB in patients with obstructive lung diseases.

Methods: The review was registered on PROSPERO (CRD42018095874). PubMed, CENTRAL, Scopus and Web of Science were searched from inception to September 2018. Papers reporting cases of incident TB in patients with obstructive lung diseases were included; studies without data on ICS use were excluded. Simultaneous use of oral corticosteroids (OCS) and population attributable fraction (PAF) for TB from ICS exposure were also assessed. Data were analyzed using a generic inverse variance method with a randomeffects model. ORs with $95 \%$ CI were estimated.

Results: Out of 4044 retrieved papers, 9 articles evaluating adult patients only were included in the review. 36,351 patients were prescribed ICS, while 147,171 were not. Any ICS use was associated with an increased risk of TB versus no ICS use (OR=1.46; 95\% CI 1.06 to $2.01 ; \mathrm{p}=0.02 ; \mathrm{I}^{2}=96 \%$ ). A similar result was also found for current ICS use versus prior/no ICS use, as well as for high, moderate and low ICS dose versus no ICS. When simultaneous OCS use was evaluated, the independent contribution of ICS was confirmed only in patients not on OCS (OR=1.63; 95\% CI 1.05 to $\left.2.52 ; \mathrm{p}=0.03 ; \mathrm{I}^{2}=94 \%\right)$. Only $0.49 \%$ of all TB cases could be attributable to ICS exposure.

Conclusions: Despite the association between ICS and TB, the contribution of this risk factor to the epidemiology of TB seems to be limited. As a consequence, no populationbased interventions are warranted. Rather, this risk should be taken into account on an individual basis, particularly in those patients with a high risk of progression from LTBI to TB.

Keywords: tuberculosis, inhaled corticosteroids, obstructive lung disease, infection control, meta-analysis

\section{Introduction}

Despite the goals achieved with the "Global Plan to Stop TB" (2006-2015), tuberculosis (TB) remains the leading cause of death from a single infectious agent, surpassing HIV/AIDS and malaria. ${ }^{1}$ The World Health Organization thus launched a new program called "The End TB Strategy" with the aims of ending the global TB epidemic by 2035 through a reduction in deaths by $95 \%$, and in 
incidence by $90 \%$ compared with levels in $2015 .^{1,2}$ In order to reach these targets, the prevention of future cases of illness through the diagnosis and treatment of latent TB infection (LTBI) has a key role. ${ }^{3,4}$ In LTBI, the subject is infected with M. tuberculosis, but does not present any clinical, microbiological and radiological evidence of active disease. If untreated, it carries a $5-10 \%$ risk in life of developing active TB in an immunocompetent subject. This risk is further increased among people belonging to specific categories, including patients on chronic steroid therapy. ${ }^{1,5-8}$

Systemic corticosteroid therapy is used in the treatment of exacerbations of asthma and COPD, and in step 5 of severe asthma. ${ }^{9,10}$ Indeed, inhaled corticosteroids (ICS) are the cornerstone of stable asthma treatment. In COPD, although their use has been progressively reduced, they are generally prescribed at higher doses by virtue of the reduced responsiveness of the majority of patients. ${ }^{9-14}$

Current available data show an association between systemic corticosteroid therapy and an increased risk of reactivation of LTBI. ${ }^{15,16}$ In relation to ICS, GOLD guidelines 2019 state that it is not possible to draw definitive conclusions, although observational studies and a metaanalysis of randomized controlled trials describe a possible association. ${ }^{10}$ It is worth noting that in the cited metaanalysis, both follow-up and number of incident cases of TB were limited. ${ }^{17}$ In order to overcome the above limitations, we performed a systematic review and meta-analysis of non-randomized trials to evaluate the effects of ICS on the risk of TB in patients with obstructive lung diseases. To further characterize the impact of ICS exposure on the epidemiology of TB, the population attributable fraction (PAF) was also estimated.

\section{Materials And Methods}

The systematic review was registered on PROSPERO (registration number CRD42018095874) and performed in accordance with the Preferred Reporting Items for Systematic Reviews and Meta-Analyses (PRISMA) statement ( $\underline{\text { Supplementary materials }}){ }^{18}$

\section{Search Strategy}

A six-step search strategy was planned. First, we searched sentinel studies in PubMed. Second, keywords and MeSH terms were identified in PubMed. Third, in order to test the strategy, the terms "tuberculosis" and "inhaled corticosteroids" (including beclomethasone, budesonide, flunisolide, fluticasone, mometasone and triamcinolone) were searched in PubMed. The full strategy can be found in the Supplementary materials. Fourth, PubMed, CENTRAL, Scopus and Web of Science were searched. Fifth, nonrandomized studies reporting cases of incident $\mathrm{TB}$ in patients with obstructive lung diseases along with data on ICS use were selected. We excluded: 1) reviews, meta-analyses and guidelines; 2) randomized trials; 3) case reports and case series; 4) studies without data on ICS use and 5) studies on non-tuberculous mycobacteria. Finally, a manual search was conducted by screening the references of included studies for additional relevant papers. Databases were searched from inception to September 23, 2018. No language restriction was adopted. Data were synthesized in a PRISMA flowchart. Two investigators (Marco Castellana, Carlo Castellana) independently and in duplicate searched papers, screened titles and abstracts of the retrieved articles, reviewed the fulltexts and selected articles for their inclusion.

\section{Data Extraction}

The following information was extracted independently and in duplicate by three investigators (Giorgio Castellana, Marco Castellana and Carlo Castellana) through a piloted form: 1) general information on the study (author, year of publication, country, study type, indication for ICS, number of patients, age and sex); 2) characteristics of ICS use (any, current, prior or no use; high, moderate or low dose) and number of patients in each category; 3) simultaneous oral corticosteroid use (OCS) and 4) adjusted OR for TB. If adjusted estimates were not reported, we used the unadjusted measures; if neither of these was available, we calculated OR with $95 \%$ CI. The main paper and supplementary data were searched; if data were missing, authors were contacted via email. Data were cross-checked, and any discrepancy was discussed.

\section{Study Quality Assessment}

The risk of bias of included observational studies was assessed independently by two reviewers (Giorgio Castellana and Marco Castellana) through National Heart, Lung, and Blood Institute Quality Assessment Tools. Retrospective cohort studies with medical record review (MRR) were evaluated according to twelve criteria as per the Observational Cohort and Cross-Sectional Studies Tool. Retrospective cohort studies with nested case-control analysis (NCC) were evaluated according to 14 criteria as per the Case-Control Studies Tool. ${ }^{19}$ 


\section{Data Analysis}

The primary outcome was the risk of $\mathrm{TB}$ occurring in patients using ICS, estimated as the OR of TB in patients on any ICS use versus no ICS. The secondary outcomes included the OR of TB based on the following comparisons: 1) patients on current ICS use versus prior or no ICS; 2) patients on high-dose ICS use versus no ICS; 3) patients on moderate-dose ICS use versus no ICS; and 4) patients on low-dose ICS use versus no ICS. Patients with a prescription within 30 days of or using inhalers until 3 months prior to the index date were classified as current. ICS dose was classified as high if $>500 \mu \mathrm{g} /$ die, medium if $250-500 \mu \mathrm{g} / \mathrm{die}$, low if $<250 \mu \mathrm{g} /$ die fluticasone propionate equivalent. ${ }^{9}$ Subgroup analyses on simultaneous OCS use, type of studies and low- versus high-incidence countries were conducted. The endpoints were analyzed with a generic inverse variance method. Heterogeneity between studies was assessed by using $\mathrm{I}^{2}$, with $50 \%$ or higher regarded as substantial. ${ }^{20}$ Publication bias was assessed with Egger's test. We also performed sensitivity analyses by removing each study in turn. All analyses were twosided and carried out using RevMan 5.3 (The Cochrane Collaboration) and Prometa 3.0 (Internovi) with a randomeffects model; $\mathrm{p}<0.05$ denoted statistical significance.

\section{Population Attributable Fraction}

According to the World Health Organization, PAF describes the contribution of a risk factor to a disease. Specifically, it is the proportional reduction in population disease that would occur if exposure to a risk factor were reduced to an alternative ideal exposure scenario. ${ }^{21}$ PAF can be calculated according to the following formula:

$$
\mathrm{PAF}=\frac{p \times(\mathrm{RR}-1)}{p \times(\mathrm{RR}-1)+1}
$$

$\mathrm{RR}$ is the relative risk and was calculated from OR according to the approach described by Wang Zhu et al: ${ }^{22}$

$$
\mathrm{RR}=\frac{\mathrm{OR}}{1-\text { risk }_{0}+\text { risk }_{0} \times \mathrm{OR}}
$$

where risk $\mathrm{k}_{0}$ is the risk of having a positive outcome in the unexposed group, estimated as the number of TB cases/ number of patients not exposed to ICS.

" $p$ " is the prevalence of the risk factor and was estimated according to the following formula:

$$
p=p_{\text {LTBI }} \times p_{\text {ICSexposure }}
$$

where $p_{\text {LTBI }}$ is the prevalence of LTBI and $p_{\text {ICSexposure }}$ is the probability of being exposed to ICS. The latter was estimated according to the following formula:

$$
p_{\text {ICSexposure }}=p_{\text {asthma on ICS }}+p_{\mathrm{COPD} \text { on ICS }}
$$

\section{Results \\ Study Characteristics}

A total of 4044 papers were found, of which 373 were from PubMed, 160 from CENTRAL, 3314 from Scopus and 197 from Web of Science. After removal of 435 duplicates, 3609 articles were analyzed for title and abstract; 3571 records were excluded (reviews, guidelines, case reports and case series, non-tuberculous mycobacteria, articles not in the field of the review, studies not in humans). The remaining 38 papers were retrieved in full text and, finally, 9 articles were included in the systematic review (Figure 1). No additional studies were retrieved after screening the references of these papers.

\section{Study Quality Assessment}

The risk of bias of the included studies is shown in e-Tables 1 and 2 . In all, statement of the study question, definition of the study population, eligibility criteria, definition of case and exposure, timing of exposure, representativeness and enrollment of patients were adequate. No sample size justification was reported. Concerning NCCs, cases and controls were matched in all, concurrent controls were reported in five papers and no information was reported on blinding of outcome assessment. ${ }^{23-27}$ Regarding MRRs, in one study, ${ }^{28}$ a follow-up ranging from 5 to 4017 days was reported, so its duration could have been not sufficient for the detection of an association between ICS and TB in all patients. In another study ${ }^{29}$ no information was reported on blinding of outcome assessment.

\section{Qualitative Analysis (Systematic Review)}

The characteristics of the 9 included articles are summarized in Table $1 .^{23-31}$ The studies were published between 2010 and 2017 and had sample sizes ranging from 554 to 54,520 patients. Seven studies were NCCs and two were MRRs. Five studies were performed in Taiwan, two in Canada, and two in South Korea. Participants were adult outpatients diagnosed with obstructive lung diseases. 183,522 patients were included, $63 \%$ were males and the weighted mean age was $64 \pm 18$ years. 36,351 were prescribed ICS, while 147,171 were not. 


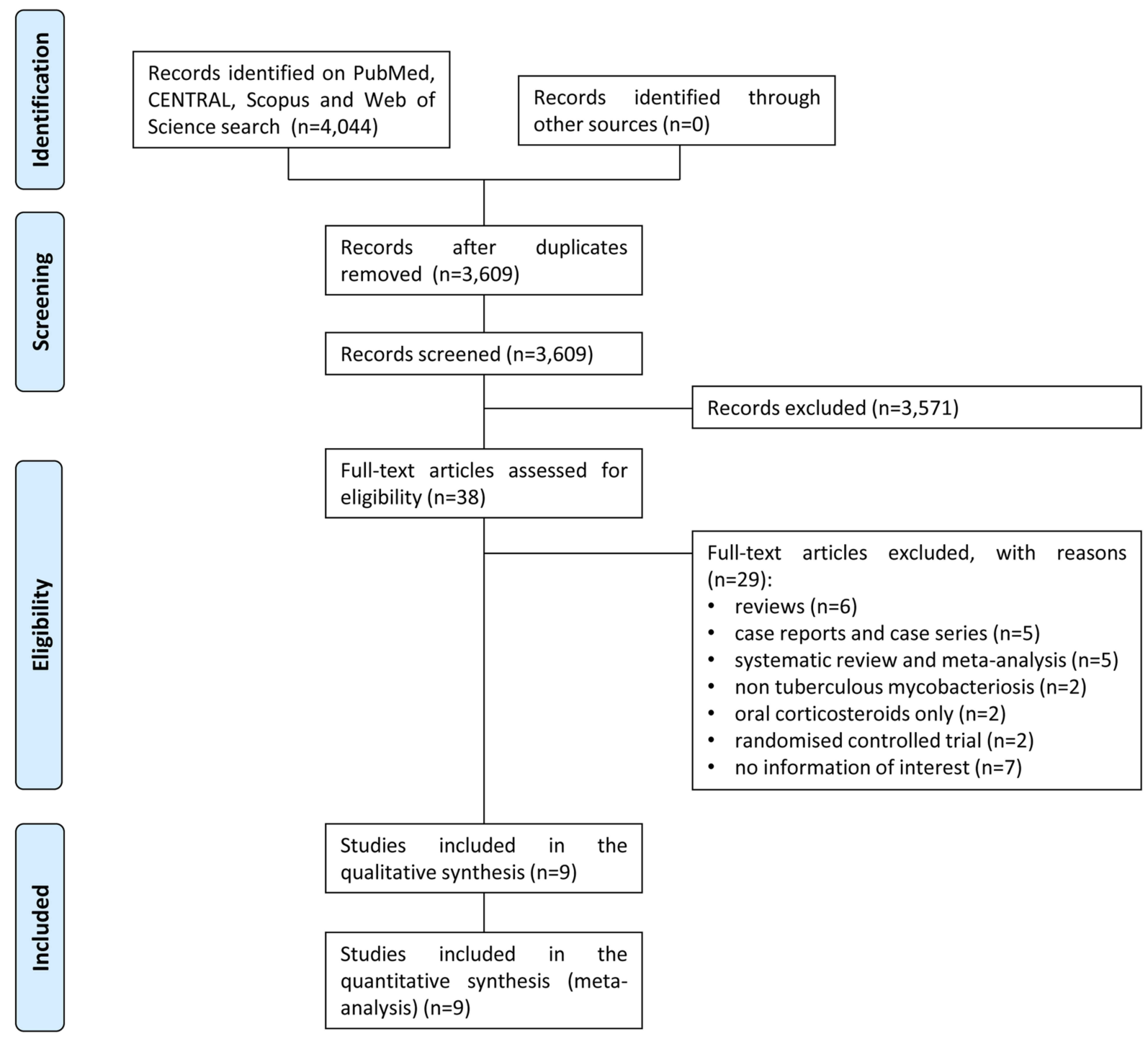

Figure I PRISMA flowchart.

\section{Quantitative Analysis (Meta-Analysis)}

Any ICS use was associated with an increased risk of TB $\left(\mathrm{OR}=1.46 ; 95 \%\right.$ CI 1.06 to $\left.2.01 ; \mathrm{p}=0.02 ; \mathrm{I}^{2}=96 \%\right)$, with MRRs showing a further increased risk $(\mathrm{OR}=4.48 ; 95 \% \mathrm{CI}$ 1.85 to $10.86 ; \mathrm{p}<0.001 ; \mathrm{I}^{2}=0 \%$ ) (Figure 2 ). When simultaneous OCS use was considered, the independent contribution of ICS was confirmed only in patients not on OCS $\left(\mathrm{OR}=1.63 ; 95 \%\right.$ CI 1.05 to $\left.2.52 ; \mathrm{p}=0.03 ; \mathrm{I}^{2}=94 \%\right)$ (Figure 3). No difference was found for low- versus high-incidence countries (e-Figure 1).

The analysis of secondary outcomes confirmed the results above. An increased risk of TB was found for current ICS use versus prior or no ICS $(\mathrm{OR}=1.25 ; 95 \%$ CI 1.13 to 1.39 ; $\mathrm{p}<0.001 ; \mathrm{I}^{2}=6 \%$ ) (e-Figure 2), high-dose ICS use versus no ICS (OR=1.99; $95 \%$ CI 1.39 to $2.87 ; \mathrm{p}<0.001 ; \mathrm{I}^{2}=75 \%$ )
(e-Figure 3), moderate-dose ICS use versus no ICS $\left(\mathrm{OR}=1.83 ; 95 \%\right.$ CI 1.48 to $\left.2.25 ; \mathrm{p}<0.001 ; \quad \mathrm{I}^{2}=0 \%\right)$ (e-Figure 4), low-dose ICS use versus no ICS (OR=2.58; $95 \%$ CI 2.02 to $3.30 ; \mathrm{p}<0.001 ; \mathrm{I}^{2}=0 \%$ ) (e-Figure 5).

There was no evidence of publication bias. In sensitivity analyses, no increased risk for any ICS use versus no ICS was found after removing five studies, each paper in turn, and for moderate dose ICS use versus no ICS after removing Chung et al, 2014 (e-Table 3) ${ }^{24}$

\section{Population Attributable Fraction}

We assumed that about $60 \%$ of patients with COPD and $100 \%$ of patients with asthma were treated with ICS. $^{32}$

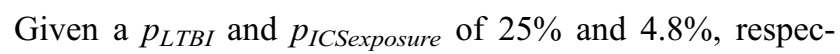
tively, the " $p$ " was estimated in $1.2 \%{ }^{33}$ We found that the 


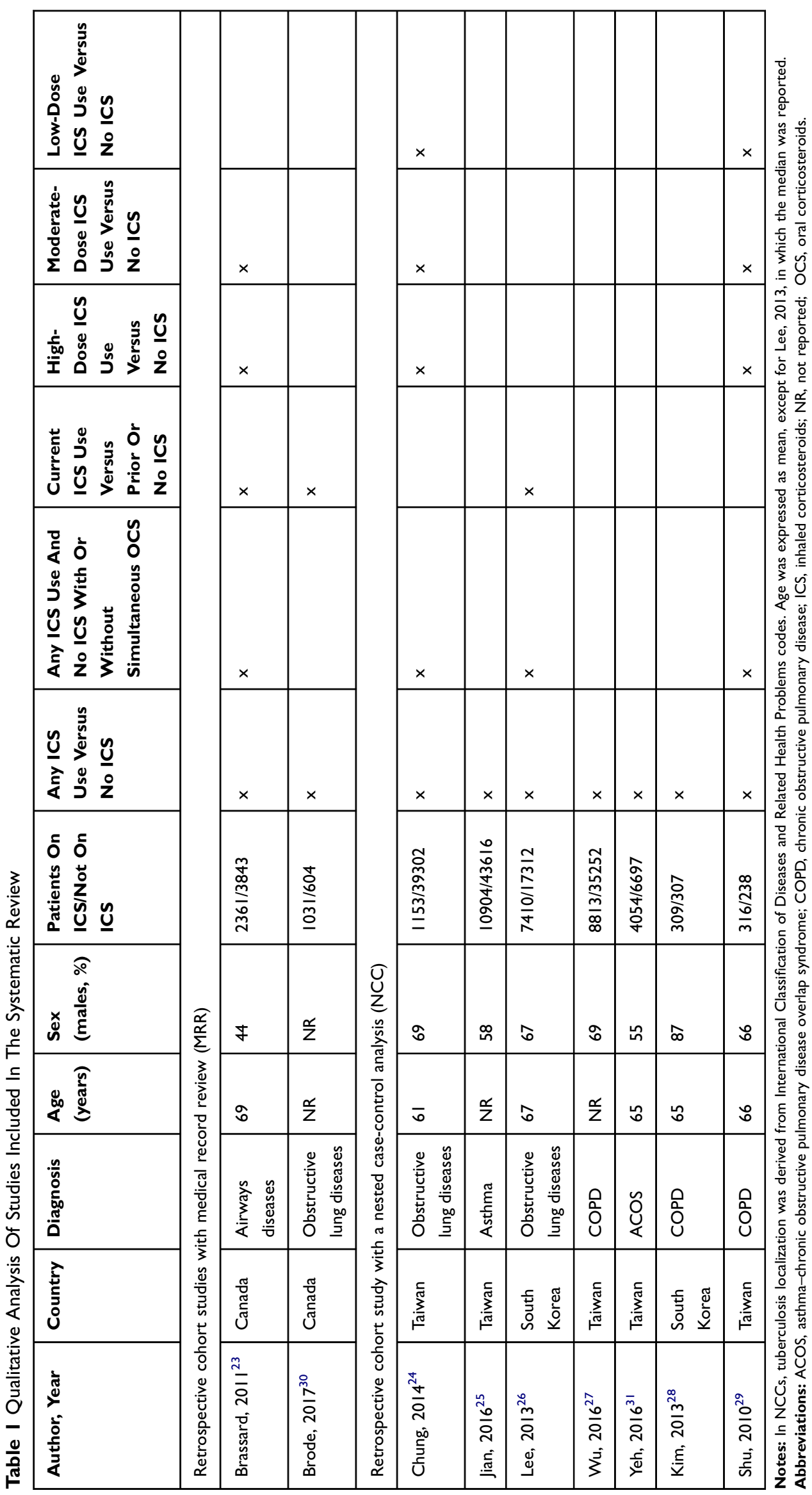




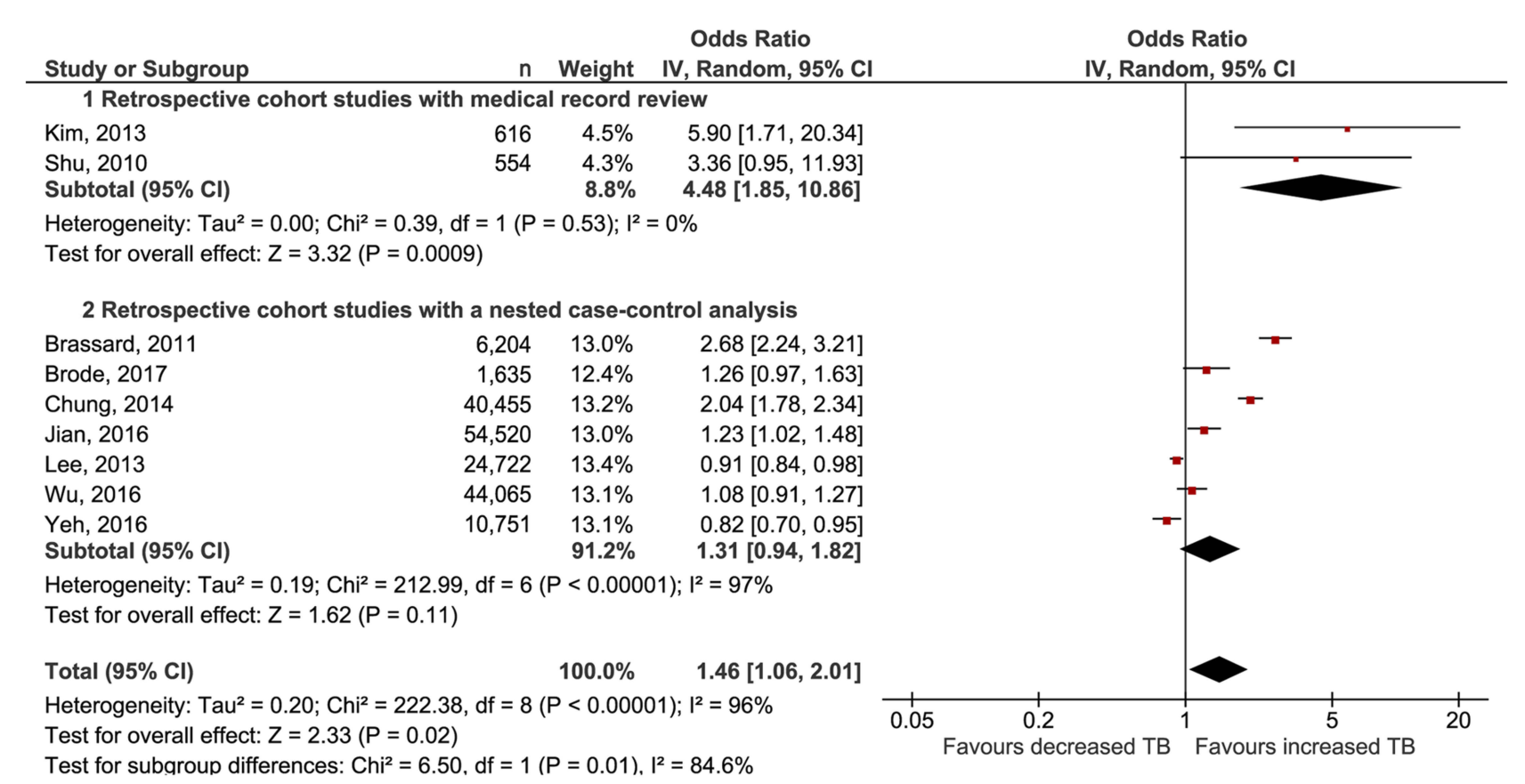

Figure 2 Forest plots of meta-analysis for difference in incident cases of TB between any ICS use and no ICS use.

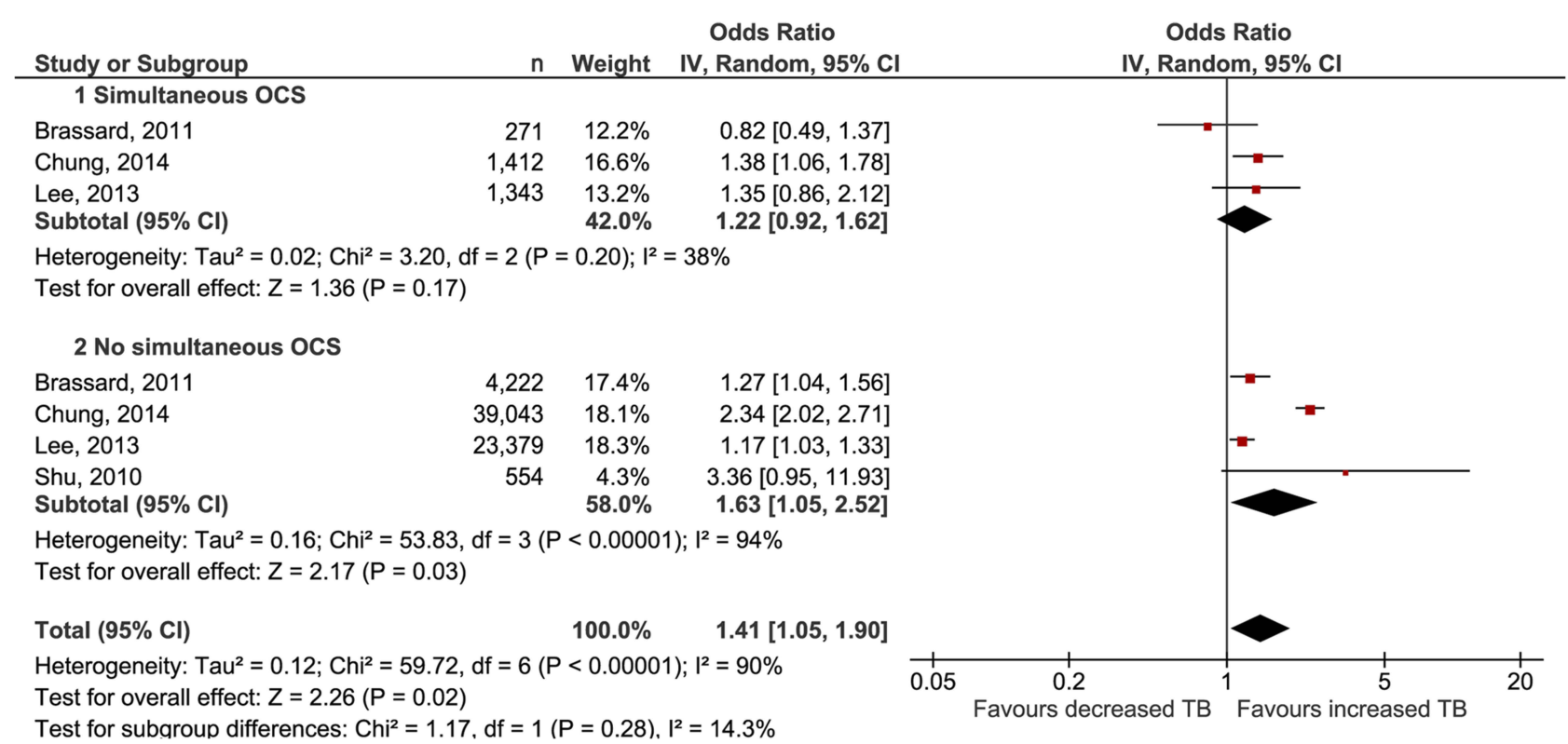

Figure 3 Forest plots of meta-analysis for difference in incident cases of TB between any ICS use and no ICS with or without simultaneous OCS.

OR for ICS was 1.46, and this corresponded to a RR of 1.41. So, PAF could be estimated in $0.49 \%$.

\section{Discussion}

The aim of this systematic review and meta-analysis was to identify the best available evidence on the impact of ICS on the risk of TB in patients with obstructive lung diseases. Overall, a positive association between any ICS use and TB was found. Comparisons on current ICS use versus prior/no ICS use, as well as on high, moderate and low ICS dose versus no ICS corroborated this finding. The independent contribution of ICS was confirmed only in patients not on OCS. A low PAF was found, meaning that the contribution of ICS exposure to the burden of TB is limited. To our knowledge, this is the first systematic review and meta-analysis assessing the impact of ICS on 
the risk of $\mathrm{TB}$ in patients with obstructive lung diseases not on OCS, as well as evaluating PAF.

According to the American Thoracic Society and the Centers for Disease Control and Prevention, corticosteroids can be associated with a progression from LTBI to TB. ${ }^{5,6}$ A case-control study conducted by Jick et al found an adjusted OR for TB of 4.9 (95\% CI 2.9-8.3) in patients on OCS, further increasing to 7.0 (95\% CI 2.9-16.8) when a $\geq 7.5 \mathrm{mg} / \mathrm{die}$ of prednisone equivalent use was considered. ${ }^{15} 750 \mu \mathrm{g} /$ die fluticasone propionate equivalent was shown to be comparable to $10 \mathrm{mg}$ of prednisone in causing adrenal suppression. ${ }^{34}$ Not surprisingly, several meta-analyses on the association between ICS use and the risk of pneumonia and upper respiratory tract infection have been published. ${ }^{35,36}$ The present review confirms that ICS can be associated with immunosuppressive effects, leading to TB. This risk may be negligible in patients on simultaneous OCS. A dose-response relationship was not found, probably meaning that even a low-dose ICS exposure may have relevant implications.

Although based on limited data, a higher OR for TB was found among MRRs compared to NCCs. In MRRs, already collected patient data are used to answer a research question. In NCCs, usually billing and hospitalization diagnosis codes are searched on health databases. Several studies reported a low accuracy for TB coding, when data were compared with patients' charts. ${ }^{37,38}$ Then, the risk of TB reported in MRRs should be more accurate than the one in NCCs.

Results were drawn from studies conducted in lowincidence as well as high-incidence countries, with rates ranging from 5/100,000/year in Canada to 70 in Republic of Korea. ${ }^{39,40}$ A comparable burden of disease is reported in the European region, with an incidence of 11/100,000/ year in the 31 European Union/European Economic area countries and of 64 in the 18 high-priority non-European Union/European Economic area countries. ${ }^{41}$ We thus believe that our results may provide useful data on the epidemiology of ICS and TB in Europe. However, no study performed in countries other than Taiwan, Canada and South Korea was found, so any inference could be highly biased.

A PAF for TB from ICS exposure of $0.49 \%$ was found. HIV, undernutrition, alcohol misuse, smoking or diabetes, which are consistently considered risk factors for TB, have a PAF of $0-70 \%, 6-63 \%, 0-35 \%, 3-29 \%$ and $2-14 \%$, respectively. ${ }^{42}$ All in all, our results indicate that there is an association between ICS use and TB, but that the impact of this risk factor on the epidemiology of TB is limited. Therefore, no population-based interventions are warranted. Rather, the practitioner should take into account this risk on an individual basis, particularly in those patients with a high risk of progression from LTBI to TB (i.e. COPD and concomitant diabetes or immunodepression). We believe that the results above do not apply to asthmatic patients having no risk factors for $\mathrm{TB}$, instead, instead, since the risk of progression is low. ${ }^{43}$

In June 2014, a meta-analysis of RCTs was published by Dong et al on ICS use and risk of TB and influenza in patients with COPD. Five studies were included; the author concluded that ICS use was associated with an increased risk of TB (OR 2.29; 95\% CI 1.04-5.03; $\left.\mathrm{p}=0.04 ; \mathrm{I}^{2}=0 \%\right)$. Of note, the follow-up was limited to 24 weeks in one study, 52 in three and 104 in one; the incident cases of TB were limited as well (18 among 5404 ICS users, 7 among 4799 non-ICS users). ${ }^{17}$ In the same month, a meta-analysis of observational studies was published by Ni et al on ICS use and risk of mycobacterial infections in patients with chronic respiratory diseases. Five studies were found, one of which was not included in the present meta-analysis because it was related to nontuberculous mycobacteria. ${ }^{44}$ ICS use was associated with an increased risk of $\mathrm{TB}$, as well $(\mathrm{RR}=1.34$; 95\% CI $1.15-$ $1.55 ; \mathrm{p}=0.001 ; \mathrm{I}^{2}=81 \%$ ), and no additional risk was found for patients on simultaneous $\mathrm{OCS}(\mathrm{RR}=1.12$; 95\% CI $\left.0.80-1.56 ; \mathrm{p}=0.53 ; \mathrm{I}^{2}=89 \%\right)$. However, no analysis was performed in patients not on OCS, so the results could have been biased by the status of the obstructive lung diseases. Moreover, analyses were performed comparing the exposure to ICS among patients with or without TB, while we evaluated the OR of TB among patients with or without ICS use. ${ }^{45}$ Due to the different approach in calculating the impact of ICS on TB, both the estimates above may be affected by a larger uncertainty than ours. Particularly, given the low number of TB cases in the former study, noise in the datasets has got eventually a higher impact on their calculated ratio. Overall, the results of our meta-analysis are consistent with these data.

Our review has several limitations. The first limitation was the database search: there may be studies published in databases other than PubMed, CENTRAL, Scopus and Web of Science. However, given the extensive search of two bibliometric databases and two citation indexes, the relevant number of retrieved records as well as the number of included patients, the possibility that studies other than the included ones may change the results of the present 
paper is low. Simultaneous OCS use was not extensively reported, and this is a second limitation. Third, we were not able to evaluate intraclass differences among ICS due to limited data. An increased incidence of pneumonia has been reported more frequently for fluticasone propionate than for budesonide, possibly due to superior duration of action and anti-inflammatory activity. ${ }^{35}$ Fourth, we were not able to perform a comparison between asthma and COPD; disease-specific data were not reported in included studies. Fifth, the substantial degree of heterogeneity for three outcomes may limit the generalizability of the results. This may be due to differences in study design, population and TB localization. Last, no study including pediatric patients was found. Further studies are thus needed.

\section{Conclusion}

In patients with obstructive lung diseases, ICS use was found to be associated with an increased risk of TB. However, when considering PAF, the contribution of ICS to the epidemiology of TB seemed to be limited. As a consequence, no population-based interventions are warranted. Rather, this risk should be taken into account on an individual basis.

\section{Acknowledgment}

The authors would like to thank Novartis Farma Italy who provided unconditional support for publishing expenses.

\section{Author Contributions}

All authors contributed to data analysis, drafting and revising the article, gave final approval of the version to be published and agree to be accountable for all aspects of the work.

\section{Disclosure}

The authors report no conflicts of interest in this work.

\section{References}

1. World Health Organization. Global Tuberculosis Report 2018; 2018. Available from: https://www.who.int/tb/publications/global_report/en/. Accessed September, 2018.

2. World Health Organization. Global strategy and targets for tuberculosis prevention, care and control after 2015; 2015. Available from: http:// www.who.int/tb/post2015_strategy/en/. Accessed September, 2018.

3. Clancy L, Rieder HL, Enarson DA, Spinaci S. Tuberculosis elimination in the countries of Europe and other industrialized countries. Eur Respir J. 1991;4(10):1288-1295.
4. Broekmans JF, Migliori GB, Rieder HL, et al. European framework for tuberculosis control and elimination in countries with a low incidence. Recommendations of the World Health Organization (WHO), International Union Against Tuberculosis and Lung Disease (IUATLD) and Royal Netherlands Tuberculosis Association (KNCV) Working Group. Eur Respir J. 2002;19(4):765-775. doi:10.1183/09031936.02.00261402

5. Ai JW, Ruan QL, Liu QH, Zhang WH. Updates on the risk factors for latent tuberculosis reactivation and their managements. Emerg Microbes Infect. 2016;5:e10. doi:10.1038/emi.2016.119

6. Lobue P, Menzies D. Treatment of latent tuberculosis infection: an update. Respirology. 2010;15(4):603-622. doi:10.1111/j.14401843.2010.01751.x

7. World Health Organization. Priorities for tuberculosis research; 2013. Available from: http://apps.who.int/iris/handle/10665/85888. Accessed September, 2018.

8. American Thoracic Society, Centers for Disease Control and Prevention. Targeted tuberculin testing and treatment of latent tuberculosis infection. Am J Respir Crit Care Med. 2000;161(4):S221-47. doi:10.1164/ajrccm.161.6.9909098

9. Global Initiative for Asthma. Global strategy for asthma management and prevention; 2018. Available from: https://ginasthma.org/2018gina-report-global-strategy-for-asthma-management-and-prevention/. Accessed, September 2018.

10. Singh D, Agusti A, Anzueto A. et al. Global strategy for the diagnosis, management, and prevention of chronic obstructive lung disease: the GOLD science committee report 2019. Eur Respir J;2019. 1900164. doi:10.1183/13993003.00164-2019

11. Suissa S, Patenaude V, Lapi F, Ernst P. Inhaled corticosteroids in COPD and the risk of serious pneumonia. Thorax. 2013;68 (11):1029-1036. doi:10.1136/thoraxjnl-2012-202872

12. Barnes PJ, Adcock IM. Glucocorticoid resistance in inflammatory diseases. Lancet. 2009;373(9678):1905-1917. doi:10.1016/S01406736(09)60326-3

13. Barnes PJ. Corticosteroid resistance in patients with asthma and chronic obstructive pulmonary disease. $J$ Allergy Clin Immunol. 2013;131(3):636-645. doi:10.1016/j.jaci.2012.12.1564

14. Barnes PJ. Inhaled corticosteroids. Pharmaceuticals (Basel). 2010;3 (3):514-540. doi:10.3390/ph3030514

15. Jick SS, Lieberman ES, Rahman MU, Choi HK. Glucocorticoid use, other associated factors, and the risk of tuberculosis. Arthritis Rheum. 2006;55(1):19-26. doi:10.1002/art.21705

16. Youssef J, Novosad SA, Winthrop KL. Infection risk and safety of corticosteroid use. Rheum Dis Clin North Am. 2016;42(1):157-176. doi:10.1016/j.rdc.2015.08.004

17. Dong YH, Chang CH, Wu FL, et al. Use of inhaled corticosteroids in patients with COPD and the risk of $\mathrm{TB}$ and influenza: a systematic review and meta-analysis of randomized controlled trials. a systematic review and meta-analysis of randomized controlled trials. Chest. 2014;145(6):1286-1297. doi:10.1378/chest.132137

18. Liberati A, Altman DG, Tetzlaff J, et al. The PRISMA statement for reporting systematic reviews and meta-analyses of studies that evaluate health care interventions: explanation and elaboration. Ann Intern Med. 2009;151(4):W65-W94. doi:10.7326/0003-4819-151-4200908180-00136

19. National Heart, Lung, and Blood Institute. Quality assessment tool for observational cohort and cross-sectional studies. quality assessment of case-control studies. Available from: https://www. nhlbi.nih.gov/health-topics/study-quality-assessment-tools. Accessed, September 2018.

20. Higgins JPT, Green S Cochrane handbook for systematic reviews of interventions version 5.1.0. The Cochrane Collaboration; 2011. [updated March, 2011]. Available from: www.handbook.cochrane. org. Accessed September 17, 2019. 
21. World Health Organization, Health statistics and information systems. Metrics: population attribuitable fraction (PAF). Available from: https://www.who.int/healthinfo/global_burden_disease/ metrics_paf/en/. Accessed February, 2019.

22. Wang Z. Converting odds ratio to relative risk in cohort studies with partial data information. J Stat Softw. 2013;55(5);1-11. doi:10.18637/ jss.v055.i05

23. Brassard P, Suissa S, Kezouh A, Ernst P. Inhaled corticosteroids and risk of tuberculosis in patients with respiratory diseases. Am J Respir Crit Care Med. 2011;183(5):675-678. doi:10.1164/rccm.201007-1099OC

24. Chung WS, Chen YF, Hsu JC, Yang WT, Chen SC, Chiang JY. Inhaled corticosteroids and the increased risk of pulmonary tuberculosis: a population-based case-control study. Int J Clin Pract. 2014;68 (10):1193-1199. doi:10.1111/ijcp.12459

25. Jian ZH, Huang JY, Lin FC, et al. Post-inhaled corticosteroid pulmonary tuberculosis increases lung cancer in patients with asthma. PLoS One. 2016;11(7):e0159683. doi:10.1371/journal.pone.0159683

26. Lee CH, Kim K, Hyun MK, Jang EJ, Lee NR, Yim JJ. Use of inhaled corticosteroids and the risk of tuberculosis. Thorax. 2013;68 (12):1105-1113. doi:10.1136/thoraxjnl-2012-203175

27. Wu MF, Jian ZH, Huang JY, et al. Post-inhaled corticosteroid pulmonary tuberculosis and pneumonia increases lung cancer in patients with COPD. BMC Cancer. 2016;16(1):778. doi:10.1186/s12885-016-2838-4

28. Kim JH, Park JS, Kim KH, Jeong HC, Kim EK, Lee JH. Inhaled corticosteroid is associated with an increased risk of TB in patients with COPD. Chest. 2013;143(4):1018-1024. doi:10.1378/chest.12-1225

29. Shu CC, Wu HD, Yu MC, et al. Use of high-dose inhaled corticosteroids is associated with pulmonary tuberculosis in patients with chronic obstructive pulmonary disease. Medicine (Baltimore). 2010;89(1):53-61. doi:10.1097/MD.0b013e3181cafcd3

30. Brode SK, Campitelli MA, Kwong JC, et al. The risk of mycobacterial infections associated with inhaled corticosteroid use. Eur Respir J. 2017;50(3):1700037. doi:10.1183/13993003.00711-2017

31. Yeh JJ, Wang YC, Kao CH. Asthma-chronic obstructive pulmonary diseases overlap syndrome increases the risk of incident tuberculosis: a national cohort study. PLoS One. 2016;11(7):e0159012. doi:10.13 71/journal.pone.0159012

32. Burgel PR, Deslée G, Jebrak G, et al. Real-life use of inhaled corticosteroids in COPD patients versus the GOLD proposals: a paradigm shift in GOLD 2011? Eur Respir J. 2014;43(4):12011203. doi:10.1183/09031936.00162313

33. James SL, Abate D, Abate KH, et al. Global, regional, and national incidence, prevalence, and years lived with disability for 354 diseases and injuries for 195 countries and territories, 1990-2017: a systematic analysis for the Global Burden of Disease Study 2017. Lancet. 2018;392(10159):1789-1858.
34. Lipworth BJ. Systemic adverse effects of inhaled corticosteroid therapy: a systematic review and meta-analysis. Arch Intern Med. 1999;159(9):941-955. doi:10.1001/archinte.159.9.941

35. Iannella H, Luna C, Waterer G. Inhaled corticosteroids and the increased risk of pneumonia: what's new? A 2015 updated review. Ther Adv Respir Dis. 2016;10(3):235-255. doi:10.1177/17534658 16630208

36. Yang M, Chen H, Zhang Y, et al. Long-term use of inhaled corticosteroids and risk of upper respiratory tract infection in chronic obstructive pulmonary disease: a meta-analysis. Inhal Toxicol. 2017;29 (5):219-226. doi:10.1080/08958378.2017.1346006

37. Campos-Outcalt DE. Accuracy of ICD-9-CM codes in identifying reportable communicable diseases. Qual Assur Util Rev. 1990;5 (3):86-89.

38. Barber C, Lacaille D, Fortin PR. Systematic review of validation studies of the use of administrative data to identify serious infections. Arthritis Care Res (Hoboken). 2013;65(8):1343-1357. doi:10.1002/ acr.21959

39. World Health Organization. Tuberculosis country profiles; 2018. Available from: http://www.who.int/tb/country/data/profiles/en/. Accessed September, 2018.

40. Centers for disease control, ministry of health and welfare, Republic of China. Taiwan. Tuberculosis Control Report 2013. April 2014. Available from: https:/www.cdc.gov.tw/english/infectionreportinfo. aspx?treeid=3847719104be0678\&nowtreeid=ffb51203f16bfe $57 \&$ tid=98263CEFADF0DAE7. Accessed September, 2018.

41. European Centre for Disease Prevention and Control/WHO Regional Office for Europe. Tuberculosis surveillance and monitoring in Europe 2018 - 2016 data. Available from: https://ecdc.europa.eu/en/ publications-data/tuberculosis-surveillance-and-monitoring-europe2018. Accessed September, 2018.

42. Lönnroth K, Castro KG, Chakaya JM, et al. Tuberculosis control and elimination 2010-50: cure, care, and social development. lancet. 2010;375(9728):1814-1829. doi:10.1016/S0140-6736(10)60483-7

43. Yii AC, Soh AZ, Chee CBE, et al. Asthma, sinonasal disease, and the risk of active tuberculosis. J Allergy Clin Immunol Pract. 2019;7 (2):641-648.e1. doi:10.1016/j.jaip.2018.07.036

44. Andréjak C, Nielsen R, Thomsen VØ, Duhaut P, Sørensen HT, Thomsen RW. Chronic respiratory disease, inhaled corticosteroids and risk of non-tuberculous mycobacteriosis. Thorax. 2013;68 (3):256-262. doi:10.1136/thoraxjnl-2012-201772

45. Ni S, Fu Z, Zhao J, Liu H. Inhaled corticosteroids (ICS) and risk of mycobacterium in patients with chronic respiratory diseases: a metaanalysis. J Thorac Dis. 2014;6(7):971-978. doi:10.3978/j.issn.20721439.2014.07.03
The International Journal of COPD is an international, peer-reviewed journal of therapeutics and pharmacology focusing on concise rapid reporting of clinical studies and reviews in COPD. Special focus is given to the pathophysiological processes underlying the disease, intervention programs, patient focused education, and self management protocols. This journal is indexed on PubMed Central, MedLine and CAS. The manuscript management system is completely online and includes a very quick and fair peer-review system, which is all easy to use. Visit http://www.dovepress.com/testimonials.php to read real quotes from published authors. 